\title{
Oscillation theorems for second-order nonlinear delay differential equations of neutral type
}

\author{
Başak Karpuz ${ }^{1}$ (D), Shyam S. Santra*2 (D) \\ ${ }^{1}$ Department of Mathematics, Faculty of Science, Tınaztepe Campus, Dokuz Eylül University, 35160 \\ Buca, Izmir, Turkey \\ ${ }^{2}$ Department of Mathematics, Sambalpur University, Sambalpur 768019, India
}

\begin{abstract}
In this paper, new sufficient conditions are obtained for oscillation of second-order neutral delay differential equations of the form

$$
\frac{\mathrm{d}}{\mathrm{d} t}\left[r(t) \frac{\mathrm{d}}{\mathrm{d} t}[x(t)+p(t) x(\tau(t))]\right]+q(t) G(x(\sigma(t)))=0 \text { for } t \geq t_{0},
$$

under the assumptions $\int^{\infty} \frac{1}{r(\eta)} \mathrm{d} \eta=\infty$ and $\int^{\infty} \frac{1}{r(\eta)} \mathrm{d} \eta<\infty$ for various ranges of the bounded neutral coefficient $p$. Unlike most of the previous results, $\tau^{\prime}$ is allowed to be oscillatory. Further, some illustrative examples showing applicability of the new results are included.
\end{abstract}

Mathematics Subject Classification (2010). 34C10, 34C15, 34K11

Keywords. Oscillation, nonoscillation, nonlinear, delay argument, second-order neutral differential equation

\section{Introduction}

This article is concerned with sufficient conditions for oscillation of a nonlinear neutral second-order delay differential equation

$$
\frac{\mathrm{d}}{\mathrm{d} t}\left[r(t) \frac{\mathrm{d}}{\mathrm{d} t}[x(t)+p(t) x(\tau(t))]\right]+q(t) G(x(\sigma(t)))=0 \text { for } t \geq t_{0} .
$$

We also suppose that the following assumptions hold:

(A1) $r \in \mathrm{C}\left(\left[t_{0}, \infty\right),(0, \infty)\right), p \in \mathrm{C}\left(\left[t_{0}, \infty\right), \mathbb{R}\right)$ and $q \in \mathrm{C}\left(\left[t_{0}, \infty\right),[0, \infty)\right)$, where $q$ is not identically zero eventually;

(A2) $G \in \mathrm{C}(\mathbb{R}, \mathbb{R})$ satisfies $u G(u)>0$ for $u \neq 0$;

(A3) $\tau, \sigma \in \mathrm{C}\left(\left[t_{0}, \infty\right), \mathbb{R}\right)$ such that $\tau(t), \sigma(t) \leq t$ for $t \geq t_{0}, \tau(t), \sigma(t) \rightarrow \infty$ as $t \rightarrow \infty$ with differentiable or invertible $\tau$ when necessary.

Our investigation on the asymptotic behavior of solutions depend on the following two possible conditions:

(C1) $\int^{\infty} \frac{1}{r(\eta)} \mathrm{d} \eta=\infty$;

(C2) $\int^{\infty} \frac{1}{r(\eta)} \mathrm{d} \eta<\infty$.

\footnotetext{
*Corresponding Author.

Email addresses: bkarpuz@gmail.com (B. Karpuz), shyam01.math@gmail.com (S.S. Santra)

Received: 20.05.2017; Accepted: 23.11.2017
} 
Baculikova et al. [1] have studied the linear counterpart of (1.1),

$$
\frac{\mathrm{d}}{\mathrm{d} t}\left[r(t) \frac{\mathrm{d}}{\mathrm{d} t}[x(t)+p(t) x(\tau(t))]\right]+q(t) x(\sigma(t))=0
$$

when $0 \leq p(t) \leq p_{0}<\infty$ and $(\mathrm{C} 1)$ hold. The authors have obtained sufficient conditions for oscillation of solutions of (1.2) through some comparison results, where the comparison results are unpredictable. In [5], Džurina have studied (1.2) when $0 \leq p(t) \leq p_{0}<\infty$ and (C1) holds true. He has established sufficient condition for oscillation of solutions of (1.2) by comparison techniques. In another paper [10], Karpuz et al. have considered the linear equation

$$
\frac{\mathrm{d}^{n}}{\mathrm{~d} t^{n}}[x(t)+p(t) x(\tau(t))]+q(t) x(\sigma(t))=0,
$$

and established some new oscillation results based on comparison theorems when $-1<$ $p(t)<0$ and $0<p(t)<1$ for all large $t$. In [15], under various ranges of $p$, Santra studied oscillatory behaviour of the solutions of the following neutral differential equations

$$
\frac{\mathrm{d}}{\mathrm{d} t}[x(t)+p(t) x(t-\tau)]+q(t) G(x(t-\sigma))=0
$$

and

$$
\frac{\mathrm{d}}{\mathrm{d} t}[x(t)+p(t) x(t-\tau)]+q(t) G(x(t-\sigma))=f(t) .
$$

Also, sufficient conditions are obtained for existence of bounded positive solutions of (1.3). The motivation of the present work come from the above studies. Hence, in this work, an attempt is made to study the more general form of (1.2) without making any comparison. It seems that our method is the next alternative to the works [1,5] and [10], when $p$ is bounded. Here, we are connected to both $(\mathrm{C} 1)$ and $(\mathrm{C} 2)$.

The neutral differential equations find numerous applications in natural sciences and technology. For instance, they are frequently used for the study of distributed networks containing lossless transmission lines (see for e.g [7]). In this paper, we restrict our attention to study (1.1), which includes a class of nonlinear functional differential equations of neutral type. In this direction we refer the reader to some of the works $([2-4,6,8,11-14,17])$ and the references cited therein.

By a solution to equation (1.1), we mean a function $x \in \mathrm{C}\left(\left[T_{x}, \infty\right), \mathbb{R}\right)$, where $T_{x} \geq t_{0}$, such that $r z^{\prime} \in \mathrm{C}^{1}\left(\left[T_{x}, \infty\right), \mathbb{R}\right)$, where

$$
z(t):=x(t)+p(t) x(\tau(t)) \text { for } t \geq T_{x},
$$

and satisfies (1.1) on the interval $\left[T_{x}, \infty\right)$. A solution $x$ of (1.1) is said to be proper if $x$ is not identically zero eventually, i.e., $\sup \{|x(t)|: t \geq T\}>0$ for all $T \geq T_{x}$. We assume that (1.1) possesses such solutions. A solution of (1.1) is called oscillatory if it has arbitrarily large zeros on $\left[T_{x}, \infty\right)$; otherwise, it is said to be nonoscillatory. (1.1) itself is said to be oscillatory if all of its solutions are oscillatory.

Remark 1.1. When the domain is not specified explicitly, all functional inequalities considered in this paper are assumed to hold eventually, i.e., they are satisfied for all $t$ large enough.

\section{Sufficient conditions for oscillation}

In this section, sufficient conditions are obtained for oscillatory and asymptotic behaviour of second order nonlinear neutral differential equations of the form (1.1). 


\subsection{Oscillation under the condition (C1)}

Lemma 2.1. Assume that (C1) and (A1)-(A3) hold. If $x$ is an eventually positive solution of (1.1) such that the companion function $z$ defined by (1.4) is also eventually positive, then $z$ satisfies

$$
z^{\prime}(t)>0 \text { and }\left(r z^{\prime}\right)^{\prime}(t)<0 \text { for all large } t .
$$

Proof. Suppose that $x(t)>0$ and $z(t)>0$ for $t \geq t_{1}$, where $t \geq t_{0}$. By (A3), we may assume without loss of generality that $x(\sigma(t))>0$ for $t \geq t_{1}$. From (1.1) and (A2), it follows that

$$
\left(r z^{\prime}\right)^{\prime}(t)=-q(t) G(x(\sigma(t)))<0 \text { for } t \geq t_{1} .
$$

Consequently, $r z^{\prime}$ is nonincreasing on $\left[t_{1}, \infty\right)$ and thus either $z^{\prime}(t)<0$ or $z^{\prime}(t)>0$ for $t \geq t_{2}$, where $t_{2} \geq t_{1}$. If $z^{\prime}(t)<0$, then there exists $\varepsilon>0$ such that $r(t) z^{\prime}(t) \leq-\varepsilon$ for $t \geq t_{2}$, which yields upon integration over $\left[t_{2}, t\right) \subset\left[t_{2}, \infty\right)$ after dividing through by $r$ that

$$
z(t) \leq z\left(t_{2}\right)-\varepsilon \int_{t_{2}}^{t} \frac{1}{r(\eta)} \mathrm{d} \eta \text { for } t \geq t_{2}
$$

In view of $(\mathrm{C} 1)$, letting $t \rightarrow \infty$ in (2.3) yields $z(t) \rightarrow-\infty$, which is a contradiction. Therefore, $z^{\prime}(t)>0$ for $t \geq t_{2}$. This completes the proof.

Remark 2.2. It follows from Lemma 2.1 that $\lim _{t \rightarrow \infty} z(t)>0$, i.e., there exists $\varepsilon>0$ such that $z(t) \geq \varepsilon$ for all large $t$.

Lemma 2.3. Assume that (C1) and (A1)-(A3) hold. If $x$ is an eventually positive solution of (1.1) such that the companion function $z$ defined by (1.4) is bounded, then $z$ satisfies (2.1) for all large $t$.

Proof. The proof can be obtained from the proof of Lemma 2.1.

Theorem 2.4. Let $0 \leq p(t) \leq p<1$ for $t \geq t_{0}$, where $p$ is a constant. Assume that (C1) and (A1)-(A3) hold. Furthermore, assume that the followings hold:

(A4) $G$ is a nondecreasing function;

(A5) $\int^{\infty} q(\eta) \mathrm{d} \eta=\infty$.

Then, every solution of (1.1) is oscillatory.

Proof. Suppose the contrary that $x$ is a nonoscillatory solution of (1.1). Then, there exists $t_{1} \geq t_{0}$ such that either $x(t)>0$ or $x(t)<0$ for $t \geq t_{1}$. Assume that $x(t)>0$, $x(\tau(t))>0$ and $x(\sigma(t))>0$ for $t \geq t_{1}$. Clearly, $z$ defined by (1.4) is positive on $\left[t_{1}, \infty\right)$. By Lemma 2.1 and Remark 2.2, there exists $\varepsilon>0$ such that $z(t) \geq \varepsilon$ for $t \geq t_{2}$, where $t_{2} \geq t_{1}$. On the other hand, $z$ being increasing implies that

$$
\begin{aligned}
(1-p) z(t) & \leq(1-p(t)) z(t) \leq z(t)-p(t) z(\tau(t)) \\
& =x(t)-p(t) p(\tau(t)) x(\tau(\tau(t))) \leq x(t)
\end{aligned}
$$

for $t \geq t_{3}$, where $t_{3} \geq t_{2}$. Consequently, $x(t) \geq(1-p) \varepsilon>0$ for $t \geq t_{3}$. From (2.2), we have

$$
\left(r z^{\prime}\right)^{\prime}(t)+G((1-p) \varepsilon) q(t) \leq 0 \text { for } t \geq t_{3} .
$$

Integrating the last inequality over the interval $\left[t_{3}, t\right) \subset\left[t_{3}, \infty\right)$, we get

$$
G((1-p) \varepsilon) \int_{t_{3}}^{t} q(\eta) \mathrm{d} \eta \leq r\left(t_{3}\right) z^{\prime}\left(t_{3}\right) \text { for all } t \geq t_{3} .
$$

This contradicts (A5).

If $x(t)<0$ for $t \geq t_{1}$, then we set $y(t):=-x(t)$ for $t \geq t_{1}$ in (1.1). Using (A4), we find

$$
\frac{\mathrm{d}}{\mathrm{d} t}\left[r(t) \frac{\mathrm{d}}{\mathrm{d} t}[y(t)+p(t) y(\tau(t))]\right]+q(t) H(y(\sigma(t)))=0 \text { for } t \geq t_{1},
$$


where $H(u):=-G(-u)$ for $u \in \mathbb{R}$. Clearly, $H$ also satisfies (A2) and (A4). Then, proceeding as above, we find the same contradiction. This completes the proof.

Theorem 2.5. Let $1 \leq p(t) \leq p$ for $t \geq t_{0}$, where $p$ is a constant. Assume that $(C 1)$ and (A1)-(A4) hold. Furthermore, assume that the following conditions hold:

(A6) there exists $\lambda>0$ such that

$$
G(u)+G(v) \geq \lambda G(u+v) \text { for } u, v \geq 0
$$

and

$$
G(u)+G(v) \leq \lambda G(u+v) \text { for } u, v \leq 0 ;
$$

and

$$
G(u v) \leq G(u) G(v) \text { for } u, v \geq 0
$$

$$
G(u v) \geq G(u) G(v) \text { for } u, v \leq 0 ;
$$

(A8) $\tau(\sigma(t))=\sigma(\tau(t))$ for $t \geq t_{0}$;

(A9) $\int^{\infty} Q(\eta) \mathrm{d} \eta=\infty$, where

$$
Q(t):=\min \left\{q(t), q(\tau(t)) \tau^{\prime}(t)\right\} \text { for } t \geq t_{0} .
$$

Then, every solution of (1.1) is oscillatory.

Proof. Without loss of generality, suppose the contrary that $x$ is an eventually positive solution of (1.1). Then, there exists $t_{1} \geq t_{0}$ such that $x(t)>0, x(\tau(t))>0$ and $x(\sigma(t))>0$ for $t \geq t_{1}$. Clearly, $z$ defined by (1.4) is positive on $\left[t_{1}, \infty\right)$. By Lemma 2.1 and Remark 2.2, there exists $\varepsilon>0$ such that $z(t) \geq \varepsilon$ for $t \geq t_{2}$, where $t_{2} \geq t_{1}$. Let us define

$$
w(t):=r(t) z^{\prime}(t)+G(p) r(\tau(t)) z^{\prime}(\tau(t))>0 \text { for } t \geq t_{3},
$$

where $t_{3} \geq t_{2}$. From (1.1), we compute that

$$
\begin{aligned}
0= & \left(r z^{\prime}\right)^{\prime}(t)+q(t) G(x(\sigma(t))) \\
& +G(p) \tau^{\prime}(t)\left[\left(r z^{\prime}\right)^{\prime}(\tau(t))+q(\tau(t)) G(x(\sigma(\tau(t))))\right] \\
= & w^{\prime}(t)+q(t) G(x(\sigma(t)))+q(\tau(t)) \tau^{\prime}(t) G(p) G(x(\tau(\sigma(t))))
\end{aligned}
$$

for $t \geq t_{4}$, where $t_{4} \geq t_{3}$. Using (A6) and (A7), we obtain

$$
\begin{aligned}
0 & \geq w^{\prime}(t)+Q(t) G(x(\sigma(t)))+G(p x(\tau(\sigma(t)))) \\
& \geq w^{\prime}(t)+\lambda Q(t) G(x(\sigma(t))+p x(\tau(\sigma(t)))) \\
& \geq w^{\prime}(t)+\lambda Q(t) G(x(\sigma(t))+p(\sigma(t)) x(\tau(\sigma(t)))) \\
& =w^{\prime}(t)+\lambda Q(t) G(z(\sigma(t)))
\end{aligned}
$$

for $t \geq t_{4}$. Consequently,

$$
w^{\prime}(t)+\lambda Q(t) G(\varepsilon) \leq 0 \text { for } t \geq t_{4},
$$

which upon integration over the interval $\left[t_{4}, t\right) \subset\left[t_{4}, \infty\right)$ yields that

$$
\lambda G(\varepsilon) \int_{t_{4}}^{t} Q(\eta) \mathrm{d} \eta \leq w\left(t_{4}\right) \text { for all } t \geq t_{4} .
$$

This contradicts (A9). Thus, $x(t)>0$ for $t \geq t_{1}$ cannot hold.

The case where $x$ is eventually negative is very similar and we omit it here. Thus, the theorem is proved.

Let us give an important example for Theorem 2.5, where the results in $[1-5,12,16]$ cannot be applied because of the limitation $\inf \left\{\tau^{\prime}(t): t \geq t_{0}\right\}>0$. Further, the results in $[8,13]$ cannot be applied either because of the nonlinear form of the delay $\tau$. 
Example 2.6. Consider the differential equation

$$
\frac{\mathrm{d}^{2}}{\mathrm{~d} t^{2}}\left[x(t)+2 x\left(t-\sin \left(\frac{\pi}{2} t\right)-1\right)\right]+x(t-4)=0 \text { for } t \geq 0,
$$

where $r(t): \equiv 1, p(t): \equiv 2, \tau(t):=t-\sin \left(\frac{\pi}{2} t\right)-1, q(t): \equiv 1, \sigma(t):=t-4$ and $G(u):=u$ for $t \geq 0$ and $u \in \mathbb{R}$. We have

$$
\tau(\sigma(t))=t-\sin \left(\frac{\pi}{2} t\right)-5=\sigma(\tau(t)) \text { and } \tau^{\prime}(t)=1-\frac{\pi}{2} \cos \left(\frac{\pi}{2} t\right) \text { for } t \geq 0 .
$$

Note that $\tau^{\prime}$ is oscillatory and $Q(t):=\min \left\{1,1-\frac{\pi}{2} \cos \left(\frac{\pi}{2} t\right)\right\}$ for $t \geq 0$. Obviously, $Q$ is a periodic function with a period of 4 . Further, $\int_{0}^{4} Q(\eta) \mathrm{d} \eta=2$, which shows that $\int^{\infty} Q(\eta) \mathrm{d} \eta=\infty$. Then, all the assumptions of Theorem 2.5 holds. Hence, every solution of $(2.5)$ oscillates.

Theorem 2.7. Let $-1 \leq p(t) \leq 0$ for $t \geq t_{0}$. Assume that (C1) and (A1)-(A5) hold. Then, every unbounded solution of (1.1) oscillates.

Proof. Without loss of generality, suppose the contrary that $x$ is an eventually positive unbounded solution of (1.1). Then, there exists $t_{1} \geq t_{0}$ such that $x(t)>0, x(\tau(t))>0$ and $x(\sigma(t))>0$ for $t \geq t_{1}$. Proceeding as in the proof of Lemma 2.1, we see $r z^{\prime}$ is nonincreasing and $z$ is monotonic on $\left[t_{2}, \infty\right)$, where $t_{2} \geq t_{1}$. We have the following two possible cases.

Case 1. Let $z(t)>0$ for $t \geq t_{2}$. By Lemma 2.1, (2.1) holds for $t \geq t_{3}$. Clearly, $z(t) \leq x(t)$ for $t \geq t_{3}$ implies

$$
\left(r z^{\prime}\right)^{\prime}(t)+q(t) G(z(\sigma(t))) \leq 0 \text { for } t \geq t_{3},
$$

where $t_{4} \geq t_{3}$. Further, by Lemma 2.1 and Remark 2.2, there exists $\varepsilon>0$ such that $z(t) \geq \varepsilon$ for $t \geq t_{4}$. Consequently, it follows from (2.6) that

$$
\left(r z^{\prime}\right)^{\prime}(t)+G(\varepsilon) q(t) \leq 0 \text { for } t \geq t_{4} .
$$

Integrating the last inequality over $\left[t_{4}, t\right) \subset\left[t_{4}, \infty\right)$, we have

$$
G(\varepsilon) \int_{t_{4}}^{t} q(\eta) \mathrm{d} \eta \leq r\left(t_{4}\right) z^{\prime}\left(t_{4}\right) \text { for } t \geq t_{4} .
$$

This contradicts (A5).

Case 2. Let $z(t)<0$ for $t \geq t_{2}$. As $x$ is unbounded, there exists $T \geq t_{2}$ such that $x(T)=$ $\max \left\{x(\eta): t_{2} \leq \eta \leq T\right\}$. Then, from (1.4), we have $x(T) \leq z(T)+x(\tau(T))<x(T)$, which is a contradiction.

The case where $x$ is an eventually negative unbounded solution is similar and we omit it here. Thus, the proof is complete.

Theorem 2.8. Let $-1<-p \leq p(t) \leq 0$ for $t \geq t_{0}$, where $p$ is a constant. Assume that (C1) and (A1)-(A5) hold. Then, every bounded solution of (1.1) either oscillates or converges to zero asymptotically.

Proof. Without loss of generality, let $x$ be an eventually positive bounded solution of (1.1). Then, there exists $t_{1} \geq t_{0}$ such that $x(t)>0, x(\tau(t))>0$ and $x(\sigma(t))>0$ for $t \geq t_{1}$. By Lemma 2.3, there exists $t_{2} \geq t_{1}$ such that $z^{\prime}(t)>0$ for $t \geq t_{2}$. Consequently, we have the following two possible cases.

Case 1. Let $z(t)>0$ for $t \geq t_{2}$. Proceeding as in Case 1 in the proof of Theorem 2.7, we get a contradiction.

Case 2. Let $z(t)<0$ for $t \geq t_{2}$. Then, $\lim _{t \rightarrow \infty} z(t)$ exits. Thus, we have

$$
\begin{aligned}
0 & \geq \lim _{t \rightarrow \infty} z(t)=\limsup _{t \rightarrow \infty} z(t)=\limsup _{t \rightarrow \infty}[x(t)+p(t) x(\tau(t))] \\
& \geq \limsup _{t \rightarrow \infty}[x(t)-p x(\tau(t))] \geq \limsup _{t \rightarrow \infty} x(t)+\liminf _{t \rightarrow \infty}[-p x(\tau(t))] \\
& =(1-p) \limsup _{t \rightarrow \infty} x(t),
\end{aligned}
$$


i.e., $\lim \sup _{t \rightarrow \infty} x(t)=0$ (since $0<p<1$ ) and hence $\lim _{t \rightarrow \infty} x(t)=0$.

The case where $x$ is an eventually negative bounded solution is omitted since it can be dealt similarly. This completes the proof.

Combining Theorem 2.5 and Theorem 2.7, we have the following corollary.

Corollary 2.9. Let $-1<-p \leq p(t) \leq 0$ for $t \geq t_{0}$, where $p$ is a constant. Assume that (C1) and (A1)-(A5) hold. Then, every solution of (1.1) either oscillates or converges to zero asymptotically.

Theorem 2.10. Let $-p_{1} \leq p(t) \leq-p_{2}<-1$ for $t \geq t_{0}$, where $p_{1}$ and $p_{2}$ are constants. Assume that (C1) and (A1)-(A5) hold. Then, every bounded solution of (1.1) either oscillates or converges to zero asymptotically.

Proof. Without loss of generality, let $x$ be an eventually positive bounded solution of (1.1). Then, $z$ defined by (1.4) is also bounded. By Lemma 2.3, $z$ is increasing. Hence, we have the following two possible cases.

Case 1. Let $z(t)>0$ for $t \geq t_{2}$. Proceeding as in Case 1 in the proof of Theorem 2.7, we get a contradiction.

Case 2. Let $z(t)<0$ for $t \geq t_{2}$. In this case, $\lim _{t \rightarrow \infty} z(t)$ exists as a nonpositive finite value. We claim that $\lim _{t \rightarrow \infty} z(t)=0$. Otherwise, $\lim _{t \rightarrow \infty} z(t)<0$, i.e., there exists $\varepsilon>0$ such that $z(t)<-\varepsilon$ for $t \geq t_{1}$. Then, we have $z(t) \geq p(t) x(\tau(t)) \geq-p_{1} x(\tau(t))$ for $t \geq t_{1}$, which implies $x(t) \geq-\frac{1}{p_{1}} z\left(\tau^{-1}(t)\right) \geq \frac{\varepsilon}{p_{1}}$ for $t \geq t_{1}$. Consequently, (2.2) becomes

$$
\left(r z^{\prime}\right)^{\prime}(t)+q(t) G\left(\frac{\varepsilon}{p_{1}}\right) \leq 0 \text { for } t \geq t_{1} .
$$

Integrating the last inequality over the interval $\left[t_{1}, t\right) \subset\left[t_{1}, \infty\right)$, we get

$$
G\left(\frac{\varepsilon}{p_{1}}\right) \int_{t_{2}}^{t} q(\eta) \mathrm{d} \eta \leq r\left(t_{2}\right) z^{\prime}\left(t_{2}\right) \text { for } t \geq t_{1} .
$$

This contradicts (A5). Therefore, $\lim _{t \rightarrow \infty} z(t)=0$. Hence,

$$
\begin{aligned}
0 & =\lim _{t \rightarrow \infty} z(t)=\liminf _{t \rightarrow \infty} z(t) \leq \liminf _{t \rightarrow \infty}\left[x(t)-p_{2} x(\tau(t))\right] \\
& \leq \limsup _{t \rightarrow \infty} x(t)+\liminf _{t \rightarrow \infty}\left[-p_{2} x(\tau(t))\right] \\
& \leq\left(1-p_{2}\right) \limsup _{t \rightarrow \infty} x(t),
\end{aligned}
$$

which implies that $\lim \sup _{t \rightarrow \infty} x(t)=0\left(\right.$ since $\left.p_{2}>1\right)$. Thus, $\liminf \inf _{t \rightarrow \infty} x(t)=0$ and hence $\lim _{t \rightarrow \infty} x(t)=0$.

Therefore, any nonoscillatory solution $x$ of (1.1) converges to zero. This completes the proof of the theorem.

Example 2.11. Consider the differential equations

$$
\frac{\mathrm{d}}{\mathrm{d} t}\left[t \frac{\mathrm{d}}{\mathrm{d} t}\left[x(t)-3 x\left(\mathrm{e}^{-\pi} t\right)\right]\right]+\frac{4}{t} x(t)=0 \text { for } t \geq 1,
$$

where $r(t):=t, p(t): \equiv-3, \tau(t):=\mathrm{e}^{-\pi} t, q(t):=\frac{4}{t}, \sigma(t):=t$ and $G(u):=u$ for $t \geq 1$ and $u \in \mathbb{R}$. It can be easily shown that Theorem 2.10 applies to (2.7). Thus, every bounded solution oscillates or converges to zero asymptotically. Obviously, $x(t)=\sin (\ln (t))$ for $t \geq 1$ is an oscillating solution. 


\subsection{Oscillation under the condition (C2)}

Remark 2.12. If we set

$$
R(t):=\int_{t}^{\infty} \frac{1}{r(\eta)} \mathrm{d} \eta \text { for } t \geq t_{0}
$$

then (C2) implies that $R(t) \rightarrow 0$ as $t \rightarrow \infty$.

Lemma 2.13. Assume that (C2) and (A1)-(A3) hold. If $x$ is an eventually positive solution of (1.1) such that the companion function $z$ defined by (1.4) is eventually decreasing and positive, then there exists $\varepsilon>0$ such that $z$ satisfies

$$
\varepsilon R(t) \leq z(t) \text { for all large } t,
$$

where $R$ is defined in (2.8).

Proof. Suppose that $x(t), z(t)>0$ and $z^{\prime}(t)<0$ for $t \geq t_{1}$, where $t \geq t_{0}$. By (A3), we may assume without loss of generality that $x(\sigma(t))>0$ for $t \geq t_{1}$. From (1.1) and (A2), we get (2.2). Consequently, $r z^{\prime}$ is nonincreasing on $\left[t_{1}, \infty\right)$. Therefore, $r(s) z^{\prime}(s) \leq r(t) z^{\prime}(t)$ for $s \geq t \geq t_{1}$, which implies

$$
z^{\prime}(s) \leq \frac{r(t) z^{\prime}(t)}{r(s)} \text { for } s \geq t \geq t_{1}
$$

Consequently,

$$
z(s) \leq z(t)+r(t) z^{\prime}(t) \int_{t}^{s} \frac{1}{r(\eta)} \mathrm{d} \eta \text { for } s \geq t \geq t_{1} .
$$

As $r z^{\prime}$ is nonincreasing, we can find a constant $\varepsilon>0$ such that $r(t) z^{\prime}(t) \leq-\varepsilon$ for $t \geq t_{1}$. As a result $z(s) \leq z(t)-\varepsilon \int_{t}^{s} \frac{1}{r(\eta)} \mathrm{d} \eta$ for $s \geq t \geq t_{1}$. By letting $s \rightarrow \infty$, we get $0 \leq z(t)-\varepsilon R(t)$ for $t \geq t_{1}$, which proves (2.9).

Theorem 2.14. Let $0 \leq p(t) \leq p$ for $t \geq t_{0}$, where $p$ is a constant. Assume that (C2), (A1)-(A4) and (A6)-(A9) hold. Further, assume

$$
\int_{t_{0}}^{\infty} \frac{1}{r(\eta)} \int_{t_{0}}^{\eta} Q(\zeta) G(\varepsilon R(\sigma(\zeta))) \mathrm{d} \zeta \mathrm{d} \eta=\infty \text { for every } \varepsilon>0
$$

and

$$
\int_{t_{0}}^{\infty} \frac{1}{r(\eta)} \int_{t_{0}}^{\eta} Q(\zeta) G(\varepsilon R(\sigma(\zeta))) \mathrm{d} \zeta \mathrm{d} \eta=-\infty \text { for every } \varepsilon<0,
$$

where $Q$ is defined in (A9).

Then, every solution of (1.1) is oscillatory.

Proof. Without loss of generality, assume the contrary that $x$ is an eventually positive solution of (1.1). Proceed as in the proof of Lemma 2.1 to obtain (2.2) for $t \geq t_{1}$, i.e., $r z^{\prime}$ is nonincreasing on $\left[t_{2}, \infty\right)$, where $t_{2} \geq t_{1}$. Recall that $z$ is positive on $\left[t_{2}, \infty\right)$. Thus, we have the following two cases.

Case 1. Let $z^{\prime}(t)>0$ for $t \geq t_{2}$. Then, we proceed as in Theorem 2.5 to get a contradiction.

Case 2. Let $z^{\prime}(t)<0$ for $t \geq t_{2}$. By Lemma 2.13, we have (2.9) for $t \geq t_{3}$, where $\varepsilon>0$ and $t_{3} \geq t_{2}$. Using (2.9) in (2.4), we have

$$
w^{\prime}(t)+\lambda Q(t) G(\varepsilon R(\sigma(t))) \leq 0 \text { for } t \geq t_{3},
$$

where $t_{3} \geq t_{2}$. Integrating the last inequality over the interval $\left[t_{3}, t\right) \subset\left[t_{3}, \infty\right)$, we obtain

$$
\lambda \int_{t_{3}}^{t} Q(\eta) G(\varepsilon R(\sigma(\eta))) \mathrm{d} \eta \leq-w(t) \leq-(1+G(p)) r(t) z^{\prime}(t),
$$


which implies

$$
\frac{\lambda}{1+G(p)} \frac{1}{r(t)} \int_{t_{3}}^{t} Q(\eta) G(\varepsilon R(\sigma(\eta))) \mathrm{d} \eta \leq-z^{\prime}(t) \text { for } t \geq t_{3} .
$$

Again integrating the last inequality over the interval $\left[t_{3}, t\right) \subset\left[t_{3}, \infty\right)$, we obtain

$$
\frac{\lambda}{1+G(p)} \int_{t_{3}}^{t} \frac{1}{r(\eta)} \int_{t_{3}}^{\eta} Q(\zeta) G(\varepsilon R(\sigma(\zeta))) \mathrm{d} \zeta \mathrm{d} \eta \leq z\left(t_{3}\right) \text { for } t \geq t_{3},
$$

which contradicts (A10).

The case where $x$ is eventually negative can be dealt similarly, and we omit the details here. This completes the proof.

Example 2.15. Consider the differential equations

$$
\frac{\mathrm{d}}{\mathrm{d} t}\left[\mathrm{e}^{t} \frac{\mathrm{d}}{\mathrm{d} t}\left[x(t)+3 \mathrm{e}^{-t} x(t-3)\right]\right]+\mathrm{e}^{3 t}(x(t-1))^{3}=0 \text { for } t \geq 3,
$$

where $r(t):=\mathrm{e}^{t}, R(t):=\mathrm{e}^{-t}, p(t):=3 \mathrm{e}^{-t}, \tau(t):=t-3, q(t):=\mathrm{e}^{3 t}, \sigma(t):=t-1$ and $G(u):=u^{3}$ for $t \geq 3$ and $u \in \mathbb{R}$. Then, all the assumptions of Theorem 2.14 holds. Hence, every solution of (2.10) oscillates.

Theorem 2.16. Let $-1 \leq p(t) \leq 0$ for $t \geq t_{0}$. Assume that (C2) and (A1)-(A5) hold. Furthermore, assume that

and

$$
\int_{t_{0}}^{\infty} \frac{1}{r(\eta)} \int_{t_{0}}^{\eta} q(\zeta) G(\varepsilon R(\sigma(\zeta))) \mathrm{d} \zeta \mathrm{d} \eta=\infty \text { for every } \varepsilon>0
$$

$$
\int_{t_{0}}^{\infty} \frac{1}{r(\eta)} \int_{t_{0}}^{\eta} q(\zeta) G(\varepsilon R(\sigma(\zeta))) \mathrm{d} \zeta \mathrm{d} \eta=-\infty \text { for every } \varepsilon<0 .
$$

Then, every unbounded solution of (1.1) oscillates.

Proof. Without loss of generality, let $x$ be an eventually positive unbounded solution of (1.1). Then, there exists $t_{1} \geq t_{0}$ such that $x(t)>0, x(\tau(t))>0$ and $x(\sigma(t))>0$ for $t \geq t_{1}$. Proceeding as in the proof of Lemma 2.1, we see that $z$ and $z^{\prime}$ are of single sign on $\left[t_{2}, \infty\right)$, where $t_{2} \geq t_{1}$. Consequently, we have the following two possible cases.

Case 1. Let $z(t)>0$ for $t \geq t_{2}$. Note that in this case, we have $z(t) \leq x(t)$ for $t \geq t_{2}$.

(a) Let $z^{\prime}(t)>0$ for $t \geq t_{2}$. We easily get (2.6). Then, proceeding as in Case 1 in the proof of Theorem 2.7, we get a contradiction.

(b) Let $z^{\prime}(t)<0$ for $t \geq t_{2}$. By Lemma 2.13, we have (2.9) for $t \geq t_{3}$, where $\varepsilon>0$ and $t_{3} \geq t_{2}$. Using $z(t) \leq x(t)$ for $t \geq t_{2}$ and $(2.2)$, we get

$$
\left(r z^{\prime}\right)^{\prime}(t)+q(t) G(\varepsilon R(\sigma(t))) \leq 0 \text { for } t \geq t_{3},
$$

where $t_{3} \geq t_{2}$. The rest of the proof follows similar to Case 2 in the proof of Theorem 2.14. Case 2. Let $z(t)<0$ for $t \geq t_{2}$. The proof is similar to Case 2 in the proof of Theorem 2.7. The proof is therefore completed.

Theorem 2.17. Let $-1<-p \leq p(t) \leq 0$ for $t \geq t_{0}$, where $p$ is a constant. Assume that (C2), (A1)-(A5) and (A11) hold. Then, every bounded solution of (1.1) either oscillates or converges to zero asymptotically.

Proof. Without loss of generality, let $x$ be an eventually positive bounded solution of (1.1). Then, there exists $t_{1} \geq t_{0}$ such that $x(t)>0, x(\tau(t))>0$ and $x(\sigma(t))>0$ for $t \geq t_{1}$. Proceeding as in the proof of Lemma 2.1, we see that $z$ and $z^{\prime}$ are of single sign on $\left[t_{2}, \infty\right)$, where $t_{2} \geq t_{1}$. Consequently, we have the following two possible cases.

Case 1. Let $z(t)>0$ for $t \geq t_{2}$. In this case, we proceed as in Case 1 in the proof of Theorem 2.16 and get a contradiction. 
Case 2. Let $z(t)<0$ for $t \geq t_{2}$. Recalling that $z$ is monotonic, we follow the steps in Case 2 in the proof of Theorem 2.8 and see that $\lim _{t \rightarrow \infty} x(t)=0$.

Hence, the proof of the theorem is complete.

Combining Theorem 2.16 and Theorem 2.17, we have the following corollary.

Corollary 2.18. Let $-1<-p \leq p(t) \leq 0$ for $t \geq t_{0}$, where $p$ is a constant. Assume that (C2), (A1)-(A5) and (A11) hold. Then, every solution of (1.1) either oscillates or converges to zero asymptotically.

Theorem 2.19. Let $-p_{1} \leq p(t) \leq-p_{2}<-1$ for $t \geq t_{0}$, where $p_{1}$ and $p_{2}$ are constants. Assume that (C2), (A1)-(A5) and (A11) hold. Further, assume that

(A12) $\int_{t_{0}}^{\infty} \frac{1}{r(\eta)} \int_{t_{0}}^{\eta} q(\zeta) \mathrm{d} \zeta \mathrm{d} \eta=\infty$.

Then, every bounded solution of (1.1) either oscillates or converges to zero asymptotically.

Proof. Without loss of generality, let $x$ be an eventually positive bounded solution of (1.1). Then, there exists $t_{1} \geq t_{0}$ such that $x(t)>0, x(\tau(t))>0$ and $x(\sigma(t))>0$ for $t \geq t_{1}$. Proceeding as in the proof of Lemma 2.1, we see that $z$ and $z^{\prime}$ are of single sign on $\left[t_{2}, \infty\right)$, where $t_{2} \geq t_{1}$. Consequently, we have the following two possible cases.

Case 1. Let $z(t)>0$ for $t \geq t_{2}$. In this case, we proceed as in Case 1 in the proof of Theorem 2.16 and get a contradiction.

Case 2. Let $z(t)<0$ for $t \geq t_{2}$. In this case, $\lim _{t \rightarrow \infty} z(t)$ exists as a nonpositive finite value. We claim that $\lim _{t \rightarrow \infty} z(t)=0$. Otherwise, $\lim _{t \rightarrow \infty} z(t)<0$, i.e., there exists $\varepsilon>0$ such that $z(t)<-\varepsilon$ for $t \geq t_{2}$. Then, we have $z(t) \geq p(t) x(\tau(t)) \geq-p_{1} x(\tau(t))$ for $t \geq t_{2}$, which implies $x(t) \geq-\frac{1}{p_{1}} z\left(\tau^{-1}(t)\right) \geq \frac{\varepsilon}{p_{1}}$ for $t \geq t_{2}$. Consequently, (2.2) becomes

$$
\left(r z^{\prime}\right)^{\prime}(t)+q(t) G\left(\frac{\varepsilon}{p_{1}}\right) \leq 0 \text { for } t \geq t_{2}
$$

Integrating the last inequality over the interval $\left[t_{2}, t\right) \subset\left[t_{2}, \infty\right)$, we get

$$
-r\left(t_{2}\right) z^{\prime}\left(t_{2}\right)+G\left(\frac{\varepsilon}{p_{1}}\right) \int_{t_{2}}^{t} q(\eta) \mathrm{d} \eta \leq-r(t) z^{\prime}(t) \text { for } t \geq t_{2} .
$$

Again, integrating the last inequality over the interval $\left[t_{2}, t\right) \subset\left[t_{2}, \infty\right)$ after dividing through by $r$, we get

$$
-r\left(t_{2}\right) z^{\prime}\left(t_{2}\right) \int_{t_{2}}^{t} \frac{1}{r(\eta)} \mathrm{d} \eta+G\left(\frac{\varepsilon}{p_{1}}\right) \int_{t_{2}}^{t} \frac{1}{r(\eta)} \int_{t_{2}}^{\eta} q(\zeta) \mathrm{d} \zeta \mathrm{d} \eta \leq-z(t) \text { for } t \geq t_{2},
$$

which contradicts (A12) by (C2). Therefore, $\lim _{t \rightarrow \infty} z(t)=0$. For the rest of the proof, we follow the steps in the last part of Case 2 of Theorem 2.10 to get $\lim _{t \rightarrow \infty} x(t)=0$.

Hence, the proof of the theorem is complete.

\section{Final comments}

In this section, we will be giving some simple remarks to conclude the paper.

Remark 3.1. In Theorem 2.4-Theorem 2.19, $G$ is allowed to be linear, sublinear or superlinear. A prototype of the function $G$ satisfying (A2), (A4), (A6) and (A7) is

$$
\left(1+\alpha|u|^{\beta}\right)|u|^{\gamma} \operatorname{sgn}(u) \text { for } u \in \mathbb{R},
$$

where $\alpha \geq 1$ or $\alpha=0$ and $\beta, \gamma>0$ are reals. For verifying (A6), we may take help of the well-known inequality (see [9, p. 292])

$$
u^{p}+v^{p} \geq h(p)(u+v)^{p} \text { for } u, v>0, \text { where } h(p):=\left\{\begin{array}{cc}
1, & 0 \leq p \leq 1, \\
\frac{1}{2^{p-1}}, & p \geq 1 .
\end{array}\right.
$$


Remark 3.2. If the nonlinear term $G$ is an odd function (presented as in Remark 3.1), it suffices to verify only the first conditions in (A6), (A7), (A10) and (A11).

Remark 3.3. The results of this paper also hold for equations of the form

$$
\frac{\mathrm{d}}{\mathrm{d} t}\left[r(t) \frac{\mathrm{d}}{\mathrm{d} t}[x(t)+p(t) x(\tau(t))]\right]+\sum_{i=1}^{m} q_{i}(t) G_{i}\left(x\left(\sigma_{i}(t)\right)\right)=0 \text { for } t \geq t_{0},
$$

where $r, p$ and $\tau$ satisfy previously mentioned assumptions and $q_{i}, G_{i}, \sigma_{i}(i=1,2, \cdots, m)$ satisfy the assumptions in (A1)-(A3). In order to extend Theorem 2.4-Theorem 2.19, we may assume that there exists an index $i$ such that $G_{i}, q_{i}, \sigma_{i}, Q_{i}$ fulfills (A4)-(A12).

We finalize the paper by presenting two examples, which show how Remark 3.3 can be applied.

Example 3.4. Consider the differential equation

$$
\frac{\mathrm{d}}{\mathrm{d} t}\left[\mathrm{e}^{-4 t} \frac{\mathrm{d}}{\mathrm{d} t}\left[x(t)-\frac{3}{\mathrm{e}^{2}} x(t-1)\right]\right]+8(x(t))^{3}+16 \mathrm{e}^{-5 t} x\left(\frac{t}{2}\right)=0 \text { for } t \geq 1,
$$

where $r(t):=\mathrm{e}^{-4 t}, p(t): \equiv-\frac{3}{\mathrm{e}^{2}}, \tau(t):=t-1, q_{1}(t): \equiv 8, \sigma_{1}(t):=t, G_{1}(u):=u^{3}$, $q_{2}(t):=16 \mathrm{e}^{-5 t}, \sigma_{2}(t)=\frac{t}{2}$ and $G_{2}(u):=u$ for $t \geq 1$ and $u \in \mathbb{R}$. All the assumptions of Corollary 2.9 can be verified with the index $i=1$. Hence, due to Remark 3.3, every solution of (3.1) oscillates or converges to zero asymptotically. Clearly $x(t)=\mathrm{e}^{-2 t}$ for $t \geq 1$ is a solution satisfying $\lim _{t \rightarrow \infty} x(t)=0$.

Example 3.5. Consider the differential equation

$$
\begin{aligned}
\frac{\mathrm{d}}{\mathrm{d} t}\left[\frac{1}{t^{2}}\right. & \left.\frac{\mathrm{d}}{\mathrm{d} t}\left[x(t)-\mathrm{e}^{-\pi} x(t-\pi)\right]\right] \\
& +4 \cosh (\pi) t\left[\mathrm{e}^{-\frac{\pi}{2}}(t+1) x\left(t-\frac{\pi}{2}\right)+x(t-\pi)\right]=0 \text { for } t \geq 2 \pi,
\end{aligned}
$$

where $r(t):=\frac{1}{t^{2}}, R(t):=\frac{1}{t}, p(t): \equiv \mathrm{e}^{-\pi}, \tau(t):=t-\pi, q_{1}(t):=4 \mathrm{e}^{-\frac{\pi}{2}} \cosh (\pi) t(t+1)$, $\sigma_{1}(t)=t-\frac{\pi}{2}, G_{1}(u):=u, q_{2}(t):=4 \cosh (\pi) t, \sigma_{2}(t):=t-\pi$ and $G_{2}(u):=u$ for $t \geq 2 \pi$ and $u \in \mathbb{R}$. All the assumptions of Corollary 2.18 can be verified with the index $i=2$. In particular, for (A11), we have

$$
\int_{2 \pi}^{\infty} \frac{1}{\eta} \int_{2 \pi}^{\eta} 4 \cosh (\pi) \zeta \frac{\varepsilon}{\zeta-\pi} \mathrm{d} \zeta \mathrm{d} \eta=\infty \text { for any } \varepsilon>0 .
$$

Hence, due to Remark 3.3, every solution of (3.2) oscillates, and such a solution is $x(t)=$ $\mathrm{e}^{t} \sin (t)$ for $t \geq 2 \pi$.

Acknowledgment. S.S. Santra is supported by the Department of Science and Technology (DST), New Delhi, India through the bank instruction order No. DST / IF140142/ 2014/140 (dated September 15th, 2014).

\section{References}

[1] B. Baculíková and J. Džurina, Oscillation theorems for second order neutral differential equations, Comput. Math. Appl. 61 (1), 94-99, 2011.

[2] B. Baculíková and J. Džurina, Oscillation theorems for second-order nonlinear neutral differential equations, Comput. Math. Appl. 62 (12), 4472-4478, 2011.

[3] B. Baculíková, T.X. Li and J. Džurina, Oscillation theorems for second order neutral differential equations, Electron. J. Qual. Theory Differ. Equ. 2011 (74), 1-13, 2011.

[4] B. Baculíková, T.X. Li and J. Džurina, Oscillation theorems for second-order superlinear neutral differential equations, Math. Slovaca, 63 (1), 123-134, 2013. 
[5] J. Džurina, Oscillation theorems for second order advanced neutral differential equations, Tatra Mt. Math. Publ. 48, 61-71, 2011.

[6] I. Györi and G. Ladas, Oscillation Theory of Delay Differential Equations with Applications, Oxford, Clarendon Press, 1991.

[7] J. Hale, Theory of Functional Differential Equations, New York, Springer-Verlag, 1977.

[8] M. Hasanbulli and Y.V. Rogovchenko, Oscillation criteria for second order nonlinear neutral differential equations, Appl. Math. Comput. 215 (12), 4392-4399, 2010.

[9] T.H. Hildebrandt, Introduction to the Theory of Integration, New York, Academic Press, 1963.

[10] B. Karpuz, Ö. Öcalan and S. Öztürk, Comparison theorems on the oscillation and asymptotic behaviour of higher-order neutral differential equations, Glasg. Math. J. 52 (1), 107-114, 2010.

[11] B. Karpuz, L.N. Padhy and R.N. Rath, Oscillation and asymptotic behaviour of a higher order neutral differential equation with positive and negative coefficients, Electron. J. Differ. Equ. 113, 15 pp., 2008.

[12] T.X. Li and Y.V. Rogovchenko, Oscillation theorems for second-order nonlinear neutral delay differential equations Abstr. Appl. Anal. Article ID: 594190, 1-5, 2014.

[13] T.X. Li, Y.V. Rogovchenko and C.H. Zhang, Oscillation results for second-order nonlinear neutral differential equations, Adv. Difference Equ. 336, 13 pp., 2013.

[14] Y.J. Liu, J.W. Zhang and J. Yan, Existence of oscillatory solutions of second order delay differential equations, J. Comput. Appl. Math. 277, 17-22, 2015.

[15] S.S. Santra, Existence of positive solution and new oscillation criteria for nonlinear first-order neutral delay differential equations, Differ. Equ. Appl. 8 (1), 33-51, 2016.

[16] S. Sun, T.X. Li, Z.L. Han and C. Zhang, On oscillation of second-order nonlinear neutral functional differential equations, Bull. Malays. Math. Sci. Soc. (2), 36 (3), 541-554, 2013.

[17] J. Yan, Existence of oscillatory solutions of forced second order delay differential equations, Appl. Math. Lett. 24 (8), 1455-1460, 2011. 